\title{
Effective and Efficient Approach for Power Reduction by Using Multi-Bit Flip-Flops
}

Ya-Ting Shyu, Jai-Ming Lin, Chun-Po Huang, Cheng-Wu Lin, Ying-Zu Lin, and Soon-Jyh Chang, Member, IEEE

\begin{abstract}
Power has become a burning issue in modern VLSI design. In modern integrated circuits, the power consumed by clocking gradually takes a dominant part. Given a design, we can reduce its power consumption by replacing some flip-flops with fewer multi-bit flip-flops. However, this procedure may affect the performance of the original circuit. Hence, the flip-flop replacement without timing and placement capacity constraints violation becomes a quite complex problem. To deal with the difficulty efficiently, we have proposed several techniques. First, we perform a co-ordinate transformation to identify those flipflops that can be merged and their legal regions. Besides, we show how to build a combination table to enumerate possible combinations of flip-flops provided by a library. Finally, we use a hierarchical way to merge flip-flops. Besides power reduction, the objective of minimizing the total wirelength is also considered. The time complexity of our algorithm is $\Theta\left(n^{1.12}\right)$ less than the empirical complexity of $\Theta\left(n^{2}\right)$. According to the experimental results, our algorithm significantly reduces clock power by 20-30\% and the running time is very short. In the largest test case, which contains 1700000 flip-flops, our algorithm only takes about $5 \mathrm{~min}$ to replace flip-flops and the power reduction can achieve $21 \%$.
\end{abstract}

Index Terms-Clock power reduction, merging, multi-bit flip-flop, replacement, wirelength.

\section{INTRODUCTION}

D UE to the popularity of portable electronic products, low power system has attracted more attention in recent years. As technology advances, an systems-on-a-chip (SoC) design can contain more and more components that lead to a higher power density. This makes power dissipation reach the limits of what packaging, cooling or other infrastructure can support. Reducing the power consumption not only can enhance battery life but also can avoid the overheating problem, which would increase the difficulty of packaging or cooling [1], [2]. Therefore, the consideration of power consumption in complex SOCs has become a big challenge to designers. Moreover, in modern VLSI designs, power consumed by clocking has taken a major part of the whole design especially for those designs using deeply scaled CMOS technologies [3]. Thus, several methodologies [4], [5] have been proposed to reduce the power consumption of clocking.

Manuscript received February 1, 2011; revised August 22, 2011; accepted February 16, 2012. Date of publication April 5, 2012; date of current version March 18, 2013. This work was supported in part by the National Science Council of Taiwan under Grant 100-2220-E-006-005.

The authors are with the Department of Electrical Engineering, National Cheng-Kung University, Tainan 70101, Taiwan (e-mail: kkttkkk@ sscas.ee.ncku.edu.tw; jmlin@ee.ncku.edu.tw; gppo@sscas.ee.ncku.edu.tw; lcw@sscas.ee.ncku.edu.tw; tibrius@gmail.com; soon@mail.ncku.edu.tw).

Color versions of one or more of the figures in this paper are available online at http://ieeexplore.ieee.org.

Digital Object Identifier 10.1109/TVLSI.2012.2190535

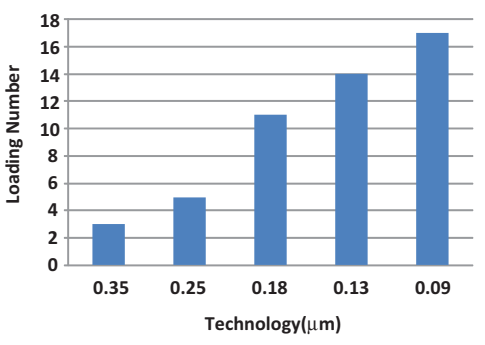

Fig. 1. Maximum loading number of a minimum-sized inverter of different technologies (rising time $250 \mathrm{ps}$ ).

Given a design that the locations of the cells have been determined, the power consumed by clocking can be reduced further by replacing several flip-flops with multi-bit flip-flops. During clock tree synthesis, less number of flip-flops means less number of clock sinks. Thus, the resulting clock network would have smaller power consumption and uses less routing resource.

Besides, once more smaller flip-flops are replaced by larger multi-bit flip-flops, device variations in the corresponding circuit can be effectively reduced. As CMOS technology progresses, the driving capability of an inverter-based clock buffer increases significantly. The driving capability of a clock buffer can be evaluated by the number of minimum-sized inverters that it can drive on a given rising or falling time. Fig. 1 shows the maximum number of minimum-sized inverters that can be driven by a clock buffer in different processes. Because of this phenomenon, several flip-flops can share a common clock buffer to avoid unnecessary power waste. Fig. 2 shows the block diagrams of 1- and 2-bit flip-flops. If we replace the two 1-bit flip-flops as shown in Fig. 2(a) by the 2-bit flip-flop as shown in Fig. 2(b), the total power consumption can be reduced because the two 1-bit flip-flops can share the same clock buffer.

However, the locations of some flip-flops would be changed after this replacement, and thus the wirelengths of nets connecting pins to a flip-flop are also changed. To avoid violating the timing constraints, we restrict that the wirelengths of nets connecting pins to a flip-flop cannot be longer than specified values after this process. Besides, to guarantee that a new flipflop can be placed within the desired region, we also need to consider the area capacity of the region. As shown in Fig. 3(a), after the two 1-bit flip-flops $f_{1}$ and $f_{2}$ are replaced by the 2-bit flip-flop $f_{3}$, the wirelengths of nets net 1 , net 2 , net 3 , and net 4 are changed. To avoid the timing violation caused by the replacement, the Manhattan distance of new nets net 1 , net $_{2}$, net 3 , and net 4 cannot be longer than the specified values. 


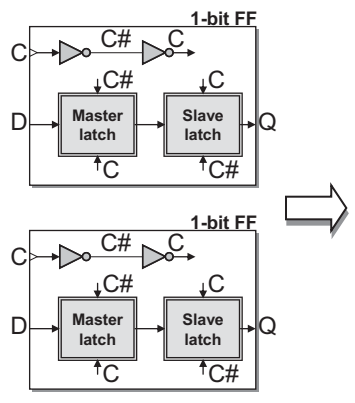

(a)

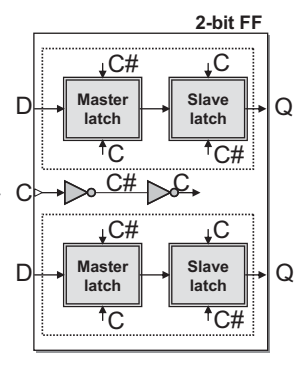

(b)
Fig. 2. Example of merging two 1-bit flip-flops into one 2-bit flip-flop. (a) Two 1-bit flip-flops (before merging). (b) 2-bit flip-flop (after merging).

In Fig. 3(b), we divide the whole placement region into several bins, and each bin has an area capacity denoting the remaining area that additional cells can be placed within it.

Suppose the area of $f_{3}$ is 7 and $f_{3}$ is assigned to be placed in the same bin as $f_{1}$. We cannot place $f_{3}$ in that bin since the remaining area of the bin is smaller than the area of $f_{3}$. In addition to the considerations mentioned in the above, we also need to check whether the cell library provides the type of the new flip-flop. For example, we have to check the availability of a 3-bit flip-flop in the cell library when we desire to replace 1- and 2-bit flip-flops by a 3-bit flip-flop.

\section{A. Related Work}

Chang et al. [6] first proposed the problem of using multi-bit flip-flops to reduce power consumption in the post-placement stage. They use the graph-based approach to deal with this problem. In a graph, each node represents a flip-flop. If two flip-flops can be replaced by a new flip-flop without violating timing and capacity constraints, they build an edge between the corresponding nodes. After the graph is built, the problem of replacement of flip-flops can be solved by finding an $m$-clique in the graph. The flip-flops corresponding to the nodes in an $m$-clique can be replaced by an $m$-bit flipflop. They use the branch-and-bound and backtracking algorithm [8] to find all $m$-cliques in a graph. Because one node (flip-flop) may belong to several $m$-cliques ( $m$-bit flip-flop), they use greedy heuristic algorithm to find the maximum independent set of cliques, which every node only belongs to one clique, while finding $m$-cliques groups. However, if some nodes correspond to $k$-bit flip-flops that $k \geqq 1$, the bit width summation of flip-flops corresponding to nodes in an $m$-clique, $j$, may not equal $m$. If the type of a $j$-bit flip-flop is not supported by the library, it may be time-wasting in finding impossible combinations of flip-flops.

\section{B. Our Contributions}

The difficulty of this problem has been illustrated in the above descriptions. To deal with this problem, the direct way is to repeatedly search a set of flip-flops that can be replaced by a new multi-bit flip-flop until none can be done. However, as the number of flip-flops in a chip increases dramatically,

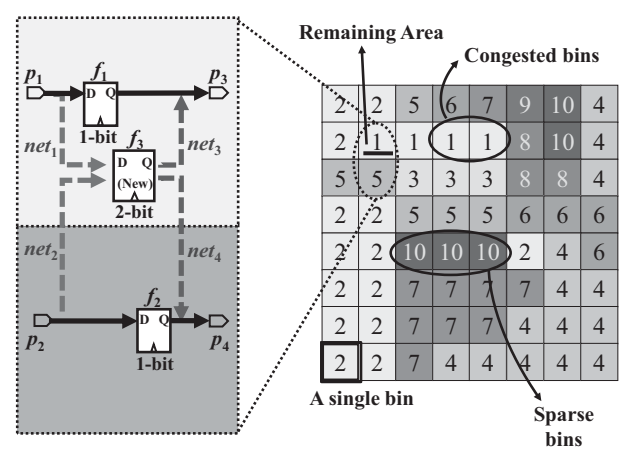

(a)

(b)

Fig. 3. (a) Combination of flip-flops possibly increases the wire length. (b) Combination of flip-flops also changes the density.

the complexity would increase exponentially, which makes the method impractical. To handle this problem more efficiently and get better results, we have used the following approaches.

1) To facilitate the identification of mergeable flip-flops, we transform the coordinate system of cells. In this way, the memory used to record the feasible placement region can also be reduced.

2) To avoid wasting time in finding impossible combinations of flip-flops, we first build a combination table before actually merging two flip-flops. For example, if a library only provides three kinds of flip-flops, which are 1-, 2-, and 3-bit, we first separate the flip-flops into three groups. Therefore, the combination of 1- and 3-bit flip-flops is not considered since the library does not provide the type of 4-bit flip-flop.

3) We partition a chip into several subregions and perform replacement in each subregion to reduce the complexity. However, this method may degrade the solution quality. To resolve the problem, we also use a hierarchical way to enhance the result.

The rest of this paper is organized as follows. Section II describes the problem formulation. Section III presents the proposed algorithm. Section IV evaluates the computation complexity. Section V shows the experimental results. Finally, we draw a conclusion in Section VI.

\section{Problem Formulation}

Before giving our problem formulation, we need the following notations.

1) Let $f_{i}$ denote a flip-flop and $b_{i}$ denote its bit width.

2) Let $A\left(f_{i}\right)$ denote the area of $f_{i}$.

3) Let $P\left(f_{i}\right)$ denote all the pins connected to $f_{i}$.

4) Let $M\left(p_{i}, f_{i}\right)$ denote the Manhattan distance between a pin $p_{i}$ and $f_{i}$, where $p_{i}$ is an $\mathrm{I} / \mathrm{O}$ pin that connects to $f_{i}$.

5) Let $S\left(p_{i}\right)$ denote the constraint of maximum wirelength for a net that connects to a pin $p_{i}$ of a flip-flop.

6) Given a placement region, we divide it into several bins [see Fig. 3(b) for example], and each bin is denoted by $B_{k}$. 


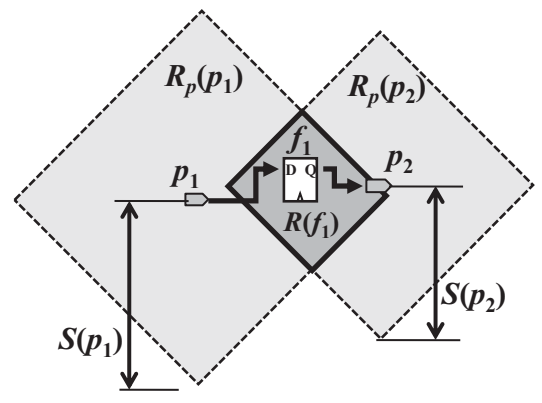

Fig. 4. Defined slack region of the pin.

7) Let $R A\left(B_{k}\right)$ denote the remaining area of the bin $B_{k}$ that can be used to place additional cells.

8) Let $L$ denote a cell library which includes different flip-flop types (i.e., the bit width or area in each type is different).

Given a cell library $L$ and a placement which contains a lot of flip-flops, our target is to merge as many flip-flops as possible in order to reduce the total power consumption. If we want to replace some flip-flops $f_{1, \ldots}, f_{j-1}$ by a new flipflop $f_{j}$, the bit width of $f_{j}$ must be equal to the summation of bit widths in the original ones (i.e., $\Sigma b_{i}=b_{j}, i=1$ to $j-1)$. Besides, since the replacement would change the routing length of the nets that connect to a flip-flop, it inevitably changes timing of some paths. Finally, to ensure that a legalized placement can be obtained after the replacement, there should exist enough space in each bin. To consider these issues, we define two constraints as follows.

1) Timing Constraint for a Net Connecting to a Flip-Flop $f_{j}$ from a Pin $p_{i}$ : To avoid that timing is affected after the replacement, the Manhattan distance between $p_{i}$ and $f_{j}$ cannot be longer than the given constraint $S\left(p_{i}\right)$ defined on the pin $p_{i}$ [i.e., $\left.M\left(p_{i}, f_{j}\right) \leq S\left(p_{i}\right)\right]$.

Based on each timing constraint defined on a pin, we can find a feasible placement region for a flip-flop $f_{j}$. See Fig. 4 for example. Assume pins $p_{1}$ and $p_{2}$ connect to a 1-bit flip-flop $f_{1}$. Because the length is measured by Manhattan distance, the feasible placement region of $f_{1}$ constrained by the pin $p_{i}$ [i.e., $\left.M\left(p_{i}, f_{1}\right) \leq S\left(p_{i}\right)\right]$ would form a diamond region, which is denoted by $R_{p}\left(p_{i}\right), i=1$ or 2 . See the region enclosed by dotted lines in the figure. Thus, the legal placement region of $f_{1}$ would be the overlapping region enclosed by solid lines, which is denoted by $R\left(f_{1}\right) . R\left(f_{1}\right)$ is the overlap region of $R_{p}\left(p_{1}\right)$ and $R_{p}\left(p_{2}\right)$.

2) Capacity Constraint for Each Bin $\boldsymbol{B}_{k}$ : The total area of flip-flops intended to be placed into the bin $B_{k}$ cannot be larger than the remaining area of the bin $B_{k}$ (i.e., $\Sigma A\left(f_{i}\right) \leq$ $\left.R A\left(B_{k}\right)\right)$.

\section{OUR Algorithm}

Our design flow can be roughly divided into three stages. Please see Fig. 5 for our flow. In the beginning, we have to identify a legal placement region for each flip-flop $f_{i}$. First, the feasible placement region of a flip-flop associated with different pins are found based on the timing constraints

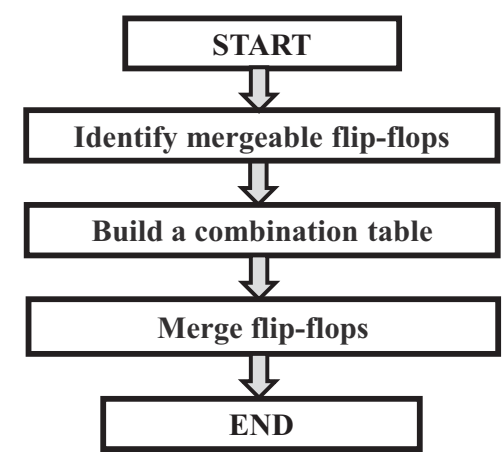

Fig. 5. Flow chart of our algorithm.

defined on the pins. Then, the legal placement region of the flip-flop $f_{i}$ can be obtained by the overlapped area of these regions. However, because these regions are in the diamond shape, it is not easy to identify the overlapped area. Therefore, the overlapped area can be identified more easily if we can transform the coordinate system of cells to get rectangular regions. In the second stage, we would like to build a combination table, which defines all possible combinations of flip-flops in order to get a new multi-bit flip-flop provided by the library. The flip-flops can be merged with the help of the table. After the legal placement regions of flip-flops are found and the combination table is built, we can use them to merge flip-flops. To speed up our program, we will divide a chip into several bins and merge flip-flops in a local bin. However, the flip-flops in different bins may be mergeable. Thus, we have to combine several bins into a larger bin and repeat this step until no flip-flop can be merged anymore.

In this section, we would detail each stage of our method. In the first subsection, we show a simple formula to transform the original coordination system into a new one so that a legal placement region for each flip-flop can be identified more easily. The second subsection presents the flow of building the combination table. Finally, the replacements of flip-flops will be described in the last subsection.

\section{A. Transformation of Placement Space}

We have shown that the shape of a feasible placement region associated with one pin $p_{i}$ connecting to a flip-flop $f_{i}$ would be diamond in Section II. Since there may exist several pins connecting to $\boldsymbol{f}_{i}$, the legal placement region of $\boldsymbol{f}_{i}$ are the overlapping area of several regions. As shown in Fig. 6(a), there are two pins $p_{1}$ and $p_{2}$ connecting to a flip-flop $f_{1}$, and the feasible placement regions for the two pins are enclosed by dotted lines, which are denoted by $R_{p}\left(p_{1}\right)$ and $R_{p}\left(p_{2}\right)$, respectively. Thus, the legal placement region $R\left(f_{1}\right)$ for $f_{1}$ is the overlapping part of these regions. In Fig. 6(b), $R\left(f_{1}\right)$ and $R\left(f_{2}\right)$ represent the legal placement regions of $f_{1}$ and $f_{2}$. Because $R\left(f_{1}\right)$ and $R\left(f_{2}\right)$ overlap, we can replace $f_{1}$ and $f_{2}$ by a new flip-flop $f_{3}$ without violating the timing constraint, as shown in Fig. 6(c).

However, it is not easy to identify and record feasible placement regions if their shapes are diamond. Moreover, four coordinates are required to record an overlapping region [see Fig. 7(a)]. Thus, if we can rotate each segment $45^{\circ}$, the 


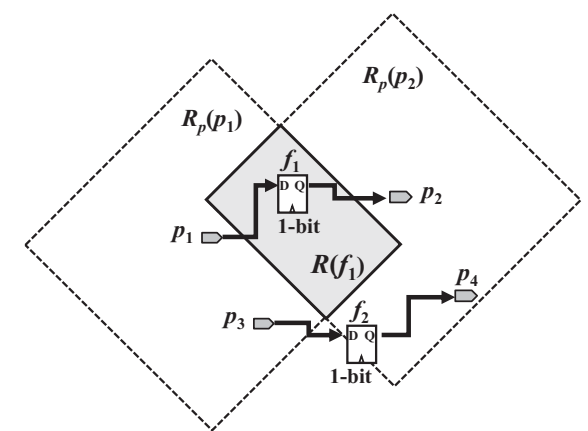

(a)

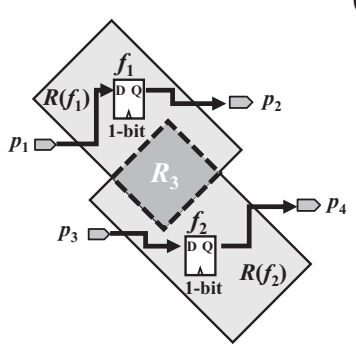

(b)

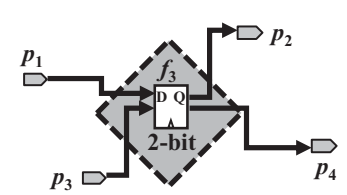

(c)
Fig. 6. (a) Feasible regions $R_{p}\left(p_{1}\right)$ and $R_{p}\left(p_{2}\right)$ for pins $p_{1}$ and $p_{2}$ which are enclosed by dotted lines, and the legal region $R\left(f_{1}\right)$ for $f_{1}$ which is enclosed by solid lines. (b) Legal placement regions $R\left(f_{1}\right)$ and $R\left(f_{2}\right)$ for $f_{1}$ and $f_{2}$, and the feasible area $R_{3}$ which is the overlap region of $R\left(f_{1}\right)$ and $R\left(f_{2}\right)$. (c) New flip-flop $f_{3}$ that can be used to replace $f_{1}$ and $f_{2}$ without violating timing constraints for all pins $p_{1}, p_{2}, p_{3}$, and $p_{4}$.

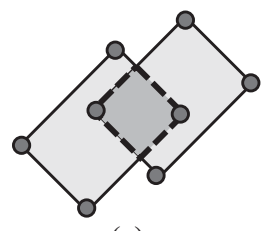

(a)

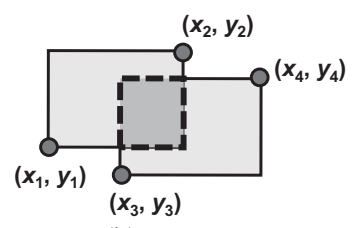

(b)
Fig. 7. (a) Overlapping region of two diamond shapes. (b) Rectangular shapes obtained by rotating the diamond shapes in (a) by $45^{\circ}$.

shapes of all regions would become rectangular, which makes identification of overlapping regions become very simple. For example, the legal placement region, enclosed by dotted lines in Fig. 7(a), can be identified more easily if we change its original coordinate system [see Fig. 7(b)]. In such condition, we only need two coordinates, which are the left-bottom corner and right-top corner of a rectangle, as shown in Fig. 7(b), to record the overlapped area instead of using four coordinates.

The equations used to transform coordinate system are shown in (1) and (2). Suppose the location of a point in the original coordinate system is denoted by $(x, y)$. After coordinate transformation, the new coordinate is denoted by $\left(x^{\prime}, y^{\prime}\right)$. In the original transformed equations, each value needs to be divided by the square root of 2 , which would induce a longer computation time. Since we only need to know the relative locations of flip-flops, such computation are ignored in our method. Thus, we use $x^{\prime \prime}$ and $y^{\prime \prime}$, to denote the coordinates of transformed locations

$$
\begin{aligned}
& x^{\prime}=\frac{x+y}{\sqrt{2}}=>x^{\prime \prime}=\sqrt{2} \cdot x^{\prime}=x+y \\
& y^{\prime}=\frac{-x+y}{\sqrt{2}}=>y^{\prime \prime}=\sqrt{2} \cdot y^{\prime}=-x+y .
\end{aligned}
$$

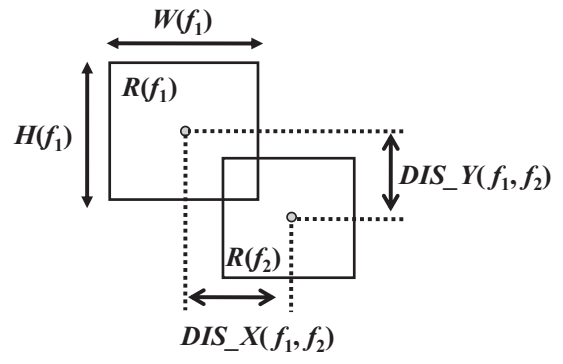

Fig. 8. Overlapping relation between available placement regions of $f_{1}$ and $f_{2}$.

Then, we can find which flip-flops are mergeable according to whether their feasible regions overlap or not. Since the feasible placement region of each flip-flop can be easily identified after the coordinate transformation, we simply use (3) and (4) to determine whether two flip-flops overlap or not

$$
\begin{aligned}
& \text { DIS_X }\left(f_{1}, f_{2}\right)<\frac{1}{2}\left(W\left(f_{1}\right)+W\left(f_{2}\right)\right) \\
& \text { DIS_Y } Y\left(f_{1}, f_{2}\right)<\frac{1}{2}\left(H\left(f_{1}\right)+H\left(f_{2}\right)\right)
\end{aligned}
$$

where $W\left(f_{1}\right)$ and $H\left(f_{1}\right)\left[W\left(f_{2}\right)\right.$ and $\left.H\left(f_{2}\right)\right]$ denote the width and height of $R\left(f_{1}\right)\left[R\left(f_{2}\right)\right]$, respectively, in Fig. 8, and the function DIS_ $X\left(f_{1}, f_{2}\right)$ and (DIS_Y $\left.\left(f_{1}, f_{2}\right)\right)$ calculates the distance between centers of $R\left(f_{1}\right)$ and $R\left(f_{2}\right)$ in $x$-direction (y-direction).

\section{B. Build a Combination Table}

If we want to replace several flip-flops by a new flip-flop $f_{i}^{\prime}$ (note that the bit width of $f_{i}^{\prime}$ should equal to the summation of bit widths of these flip-flops), we have to make sure that the new flip-flop $f_{i}^{\prime}$ is provided by the library $\boldsymbol{L}$ when the feasible regions of these flip-flops overlap. In this paper, we will build a combination table, which records all possible combinations of flip-flops to get feasible flip-flops before replacements. Thus, we can gradually replace flip-flops according to the order of the combinations of flip-flops in this table. Since only one combination of flip-flops needs to be considered in each time, the search time can be reduced greatly. In this subsection, we illustrate how to build a combination table.

The pseudo code for building a combination table $\boldsymbol{T}$ is shown in Algorithm 1. We use a binary tree to represent one combination for simplicity. Each node in the tree denotes one type of a flip-flop in $\boldsymbol{L}$. The types of flip-flops denoted by leaves will constitute the type of the flip-flop in the root. For each node, the bit width of the corresponding flip-flop equals to the bit width summation of flip-flops denoted by its left and right child [please see Fig. 9(e) for example]. Let $n_{i}$ denote one combination in $\boldsymbol{T}$, and $b\left(n_{i}\right)$ denote its bit width. In the beginning, we initialize a combination $n_{i}$ for each kind of flip-flops in $\boldsymbol{L}$ (see Line 1). Then, in order to represent all combinations by using a binary tree, we may add pseudo types, which denote those flip-flops that are not provided by the library, (see Line 2). For example, assume that a library only supports two kinds of flip-flops whose bit widths are 1 and 4 , respectively. In order to use a binary tree to denote a 


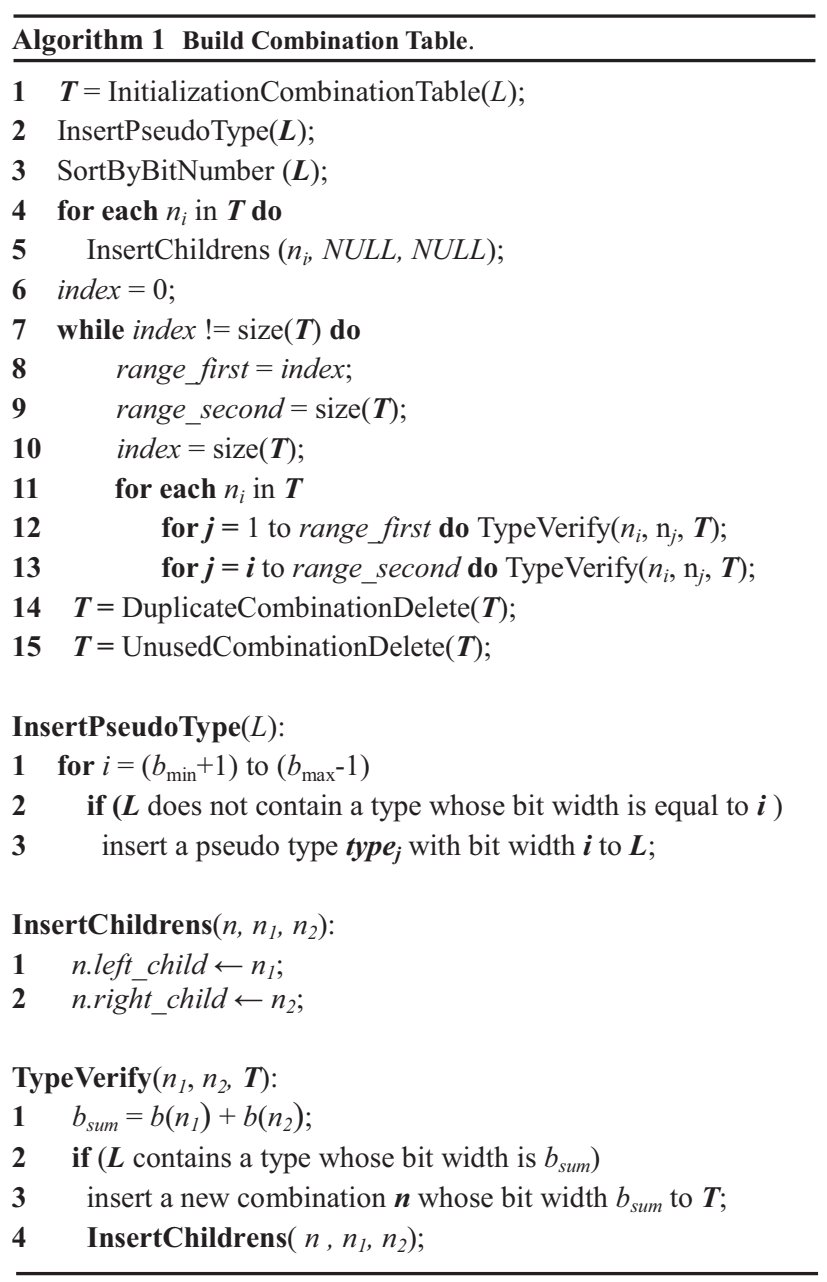

combination whose bit width is 4 , there must exist flip-flops whose bit widths are 2 and 3 in $\boldsymbol{L}$ [please see the last two binary trees in Fig. 9(e) for example]. Thus, we have to create two pseudo types of flip-flops with 2- and 3-bit if $\boldsymbol{L}$ does not provide these flip-flops. Function InsertPseudoType in algorithm 1 shows how to create pseudo types. Let $b_{\max }$ and $b_{\text {min }}$ denote the maximum and minimum bit width of flip-flops in $\boldsymbol{L}$. In InsertPseudoType, it inserts all flip-flops whose bit widths are larger than $b_{\min }$ and smaller than $b_{\max }$ into $\boldsymbol{L}$ if they are not provided by $\boldsymbol{L}$ originally. After this procedure, all combinations in $\boldsymbol{L}$ are sorted according to their bit widths in the ascending order (Line 3). At present, all combinations are represented by binary trees with 0 -level. Thus, we would assign NULL to its right and left child (see Lines 4 and 5). Finally, for every two kinds of combinations in $\boldsymbol{T}$, we try to combine them to create a new combination (Lines 6-13). If the new combination is the flip-flop of a feasible type (this can be checked by the function TypeVerify), we would add it to the table $\boldsymbol{T}$. In the function TypeVerify, we first add the bit widths of the two combinations together and store the result in $b_{\text {sum }}$ (see Line 1 in TypeVerify). Then, we will add a new combination $n$ to $\boldsymbol{T}$ with bit width $b_{\text {sum }}$ if $\boldsymbol{L}$ has such kind of a flip-flop. After these procedures, there may exist some duplicated or unused combinations in $\boldsymbol{T}$. Thus, we have

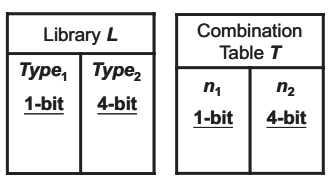

(a)
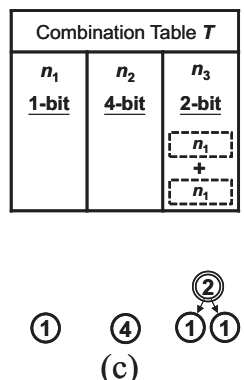

(c)

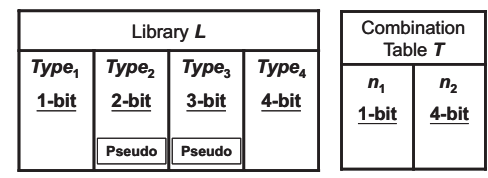

(b)

(4)

\begin{tabular}{|c|c|c|c|c|c|}
\hline \multicolumn{6}{|c|}{ Combination Table $\boldsymbol{T}$} \\
\hline$n_{1}$ & $n_{2}$ & $n_{3}$ & $n_{4}$ & $\boldsymbol{n}_{\mathbf{5}}$ & $n_{6}$ \\
\hline 1-bit & 4-bit & 2-bit & 3-bit & 4-bit & 4-bit \\
\hline & & $n_{1}$ & $n_{1}$ & $n_{3}$ & $n_{1}$ \\
\hline & & \pm & \pm & \pm & \pm \\
\hline & & $=-n_{1}$ & $-n_{3}$ & $-n_{3}-$ & $n_{4}$ \\
\hline
\end{tabular}

(4)

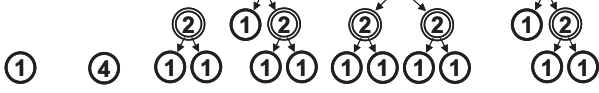

(e)

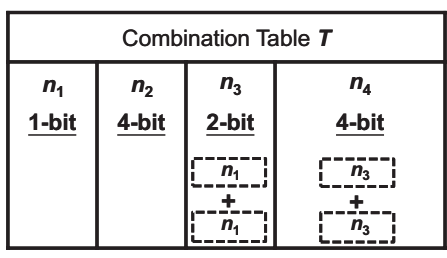

(4)

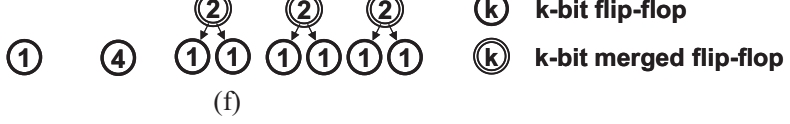

Fig. 9. Example of building the combination table. (a) Initialize the library $L$ and the combination table $T$. (b) Pseudo types are added into $L$, and the corresponding binary tree is also build for each combination in $T$. (c) New combination $n_{3}$ is obtained from combining two $n_{1} \mathrm{~s}$. (d) New combination $n_{4}$ is obtained from combining $n_{1}$ and $n_{3}$, and the combination $n_{5}$ is obtained from combining two $n_{3}$ s. (e) New combination $n_{6}$ is obtained from combining $n_{1}$ and $n_{4}$. (f) Last combination table is obtained after deleting the unused combination in (e).

to delete them from the table and the two functions DuplicateCombinationDelete and UnusedCombinationDelete are called for the purpose (Lines 14 and 15). In DuplicateCombinationDelete, it checks whether the duplicated combinations exist or not. If the duplicated combinations exist, only the one with the smallest height of its corresponding binary tree is left and the others are deleted. In UnusedCombinationDelete, it checks the combinations whose corresponding type is pseudo 


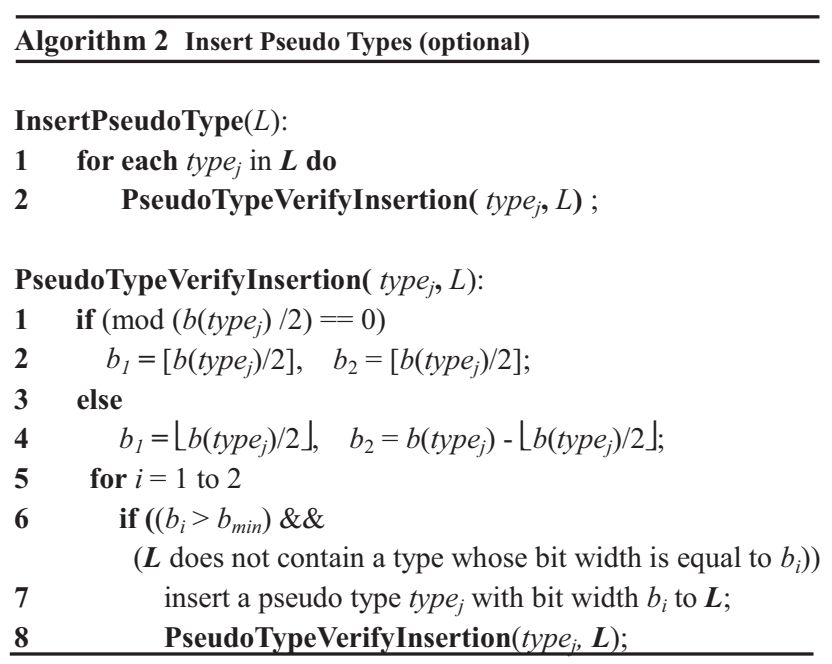

type in $\boldsymbol{L}$. If the combination is not included into any other combinations, it will be deleted.

For example, suppose a library $\boldsymbol{L}$ only provides two types of flip-flops, whose bit widths are 1 and 4 (i.e., $b_{\min }=1$ and $b_{\max }=4$ ), in Fig. 9(a). We first initialize two combinations $n_{1}$ and $n_{2}$ to represent these two types of flip-flops in the table $\boldsymbol{T}$ [see Fig. 9(a)]. Next, the function InsertPseudoType is performed to check whether the flip-flop types with bit widths between 1 and 4 exist or not. Thus, two kinds of flip-flop types whose bit widths are 2 and 3 are added into $\boldsymbol{L}$, and all types of flip-flops in $\boldsymbol{L}$ are sorted according to their bit widths [see Fig. 9(b)]. Now, for each combination in $\boldsymbol{T}$, we would build a binary tree with 0-level, and the root of the binary tree denotes the combination. Next, we try to build new legal combinations according to the present combinations. By combing two 1-bit flip-flops in the first combination, a new combination $n_{3}$ can be obtained [see Fig. 9(c)]. Similarly, we can get a new combination $n_{4}\left(n_{5}\right)$ by combining $n_{1}$ and $n_{3}$ (two $n_{3}$ 's) [see Fig. 9(d)]. Finally, $n_{6}$ is obtained by combing $n_{1}$ and $n_{4}$. All possible combinations of flip-flops are shown in Fig. 9(e). Among these combinations, $n_{5}$ and $n_{6}$ are duplicated since they both represent the same condition, which replaces four 1-bit flip-flops by a 4-bit flip-flop. To speed up our program, $n_{6}$ is deleted from $\boldsymbol{T}$ rather than $n_{5}$ because its height is larger. After this procedure, $n_{4}$ becomes an unused combination [see Fig. 9(e)] since the root of binary tree of $n_{4}$ corresponds to the pseudo type, type 3 , in $\boldsymbol{L}$ and it is only included in $n_{6}$. After deleting $n_{6}, n_{4}$ is also need to be deleted. The last combination table $\boldsymbol{T}$ is shown in Fig. 9(f).

In order to enumerate all possible combinations in the combination table, all the flip-flops whose bit widths range between $b_{\max }$ and $b_{\min }$ and do not exist in $\boldsymbol{L}$ should be inserted into $\boldsymbol{L}$ in the above procedure. However, this is time consuming. To improve the running time, only some types of flip-flops need to be inserted. There exist several choices if we want to build a binary tree corresponding to a type type ${ }_{j}$. However, the complete binary tree has the smallest height. Thus, for building a binary tree of a certain combination $n_{i}$ whose type is type $e_{j}$, only the flip-flops whose bit widths

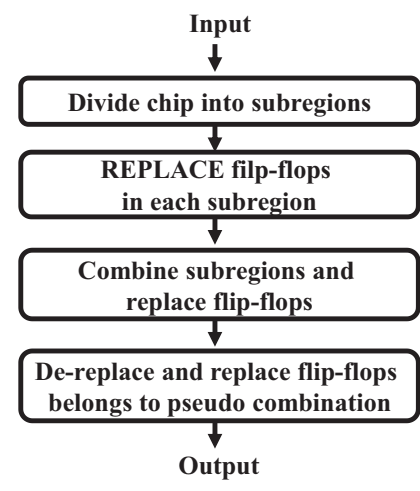

Fig. 10. Detailed flow to merge flip-flops.

are $\left(\left\lfloor b\left(\right.\right.\right.$ type $\left.\left.\left._{j}\right) / 2\right\rfloor\right)$ and $\left(b\left(\right.\right.$ type $\left._{j}\right)-\left\lfloor b\left(\right.\right.$ type $\left.\left.\left._{j}\right) / 2\right\rfloor\right)$ should exist in $\boldsymbol{L}$. Algorithm 2 shows the enhanced procedure to insert flip-flops of pseudo types. For each type $e_{j}$ in $\boldsymbol{L}$, the function PseudoTypeVerifyInsertion recursively checks the existence of flip-flops whose bit widths around $\left\lfloor b\left(\right.\right.$ type $\left.\left._{j}\right) / 2\right\rfloor$ and add them into $\boldsymbol{L}$ if they do not exist (see Lines 1 and 2). In the function PseudoTypeVerifyInsertion, it divides the bit width $b\left(\right.$ type $\left._{j}\right)$ into two parts $\left\lfloor b\left(\right.\right.$ type $\left.\left._{j}\right) / 2\right\rfloor$ and $\left\lfloor b\left(\right.\right.$ type $\left.\left._{j}\right) / 2\right\rfloor$ $\left(\left\lfloor b\left(\right.\right.\right.$ type $\left.\left._{j}\right) / 2\right\rfloor$ and $b\left(\right.$ type $\left._{j}\right)-\left\lfloor b\left(\right.\right.$ type $\left.\left.\left._{j}\right) / 2\right\rfloor\right)$ if $b\left(\right.$ type $\left._{j}\right)$ is an even (odd) number (see Lines 1-4 in PseudoTypeVerifyInsertion), and it would insert a pseudo type type $e_{j}$ into $\boldsymbol{L}$ if the type is not provided by $\boldsymbol{L}$ and its bit width is larger than the minimum bit width (denoted by $b_{\min }$ ) of flip-flops in $\boldsymbol{L}$ (see Lines $5-8$ in PseudoTypeVerifyInsertion). The same procedure repeats in the new created type. Note that this method works only when the 1-bit type exists in $\boldsymbol{L}$. We still have to insert pseudo flip-flops by the function InsertPseudoType in Algorithm 1 if the 1-bit flip-flop is not provided by $\boldsymbol{L}$.

For example, assume a library $\boldsymbol{L}$ only provides two kinds of flip-flops whose bit widths are 1 and 7 . In the new procedure, it first adds two pseudo types of flip-flops whose bit widths are 3 and 4 , respectively, for the flip-flop with 7-bit (i.e., $\boldsymbol{L}$ becomes $\left.\left[\begin{array}{llll}1 & 3 & 4 & 7\end{array}\right]\right)$. Next, the flip-flop whose bit width is 2 is added to $\boldsymbol{L}$ for the flip-flop with 4-bit (i.e., $\boldsymbol{L}$ becomes $\left.\left[\begin{array}{lllll}1 & 2 & 3 & 4 & 7\end{array}\right]\right)$. For the flip-flop with 3-bit, the procedure stops because flop-flops with 1 and 2 bits already exist in $\boldsymbol{L}$. In the new procedure, we do not need to insert 5- and 6-bit pseudo types to $\boldsymbol{L}$.

\section{Merge Flip-Flops}

We have shown how to build a combination table in Section III-B. Now, we would like to show how to use the combination table to combine flip-flops in this subsection. To reduce the complexity, we first divide the whole placement region into several subregions, and use the combination table to replace flip-flops in each subregion. Then, several subregions are combined into a larger subregion and the flip-flops are replaced again so that those flip-flops in the neighboring subregions can be replaced further. Finally, those flip-flops with pseudo types are deleted in the last stage because they are not provided by the supported library. Fig. 10 shows this flow.

1) Region Partition (Optional): To speed up our problem, we divide the whole chip into several subregions. By suitable 


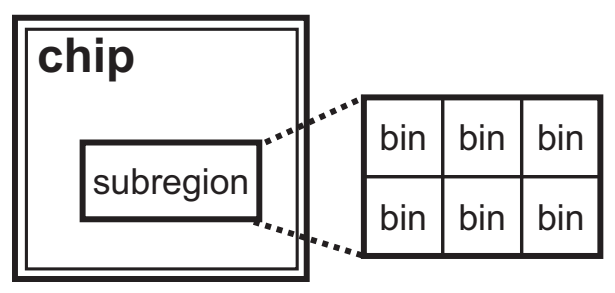

Fig. 11. Example of region partition with six bins in one subregion.

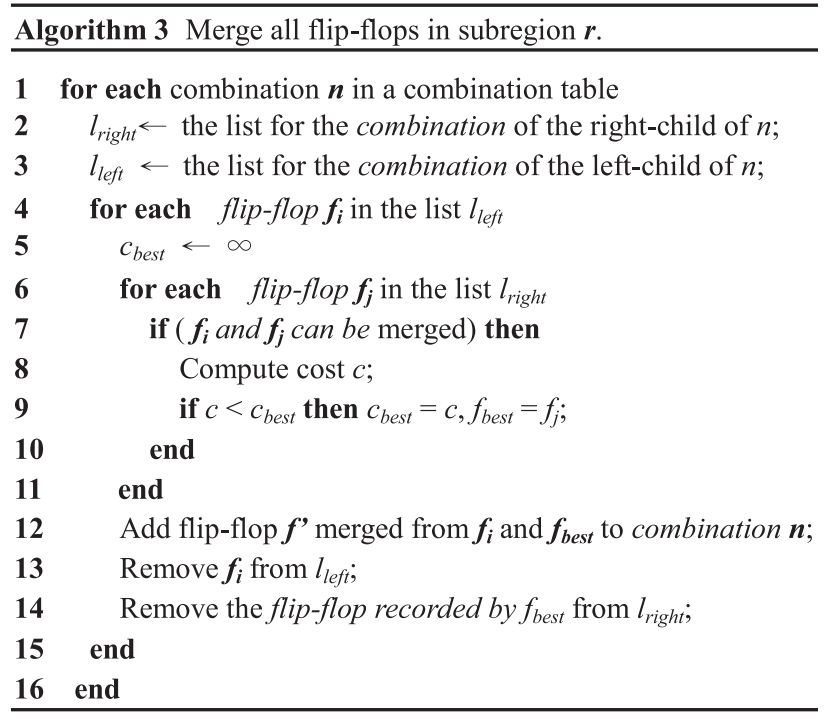

partition, the computation complexity of merging flip-flops can be reduced significantly (the related quantitative analysis will be shown in Section V). As shown in Fig. 11, we divide the region into several subregions, and each subregion contains six bins, where a bin is the smallest unit of a subregion.

2) Replacement of Flip-flops in Each Subregion: Before illustrating our procedure to merge flip-flops, we first give an equation to measure the quality if two flip-flops are going to be replaced by a new flip-flop as follows:

$$
\text { cost }=\text { routing_length }-\alpha \times \sqrt{\text { available_area }}
$$

where routing_length denotes the total routing length between the new flip-flop and the pins connected to it, and available_area represents the available area in the feasible region for placing the new flip-flop. $\alpha$ is a weighting factor (the related analysis of the value $\alpha$ will be shown in Section V). The cost function includes the term routing_length to favor a replacement that induces shorter wirelength. Besides, if the region has larger available space to place a new flip-flop, it implies that it has higher opportunities to combine with other flip-flops in the future and more power reduction. Thus, we will give it a smaller cost. Once the flip-flops cannot be merged to a higher-bit type (as the 4-bit combination $n_{4}$ in Fig. 9), we ignore the available_area in the cost function, and hence $\alpha$ is set to 0 .

After a combination has been built, we will do the replacements of flip-flops according to the combination table. First, we link flip-flops below the combinations corresponding to

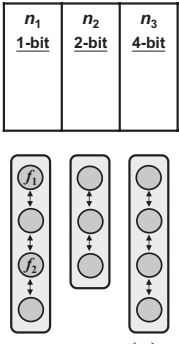

(a)

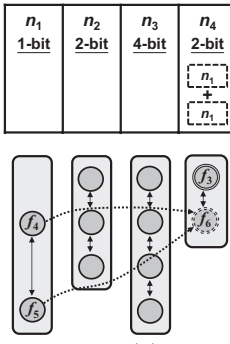

(c)
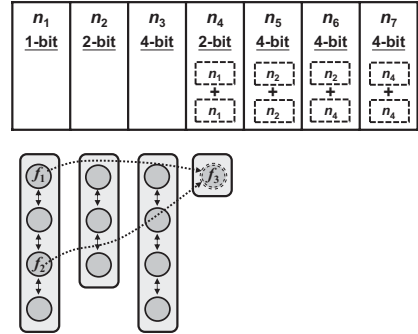

(b) (e)

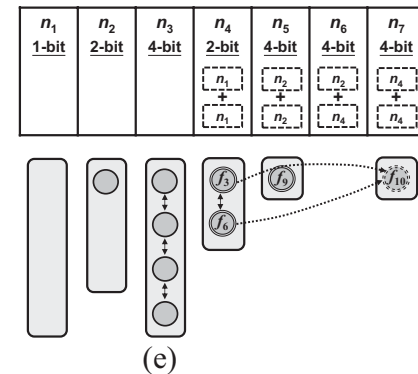

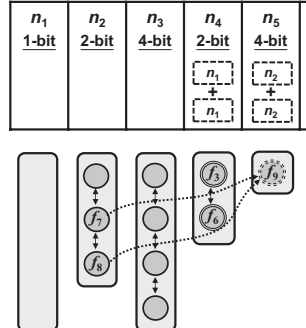

(d)

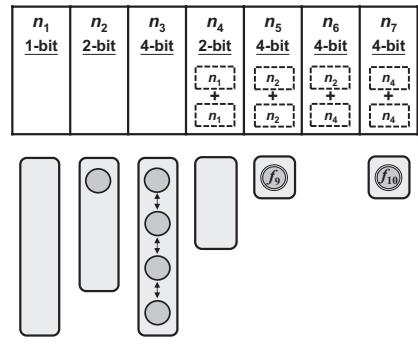

(f)
Fig. 12. Example of replacements of flip-flops. (a) Sets of flip-flops before merging. (b) Two 1-bit flip-flops, $f_{1}$ and $f_{2}$, are replaced by the 2-bit flip-flop $f_{3}$. (c) Two 1-bit flip-flops, $f_{4}$ and $f_{5}$, are replaced by the 2-bit flip-flop $f_{6}$. (d) Two 2-bit flip-flops, $f_{7}$ and $f_{8}$, are replaced by the 4-bit flip-flop $f_{9}$. (e) Two 2-bit flip-flops, $f_{3}$ and $f_{6}$, are replaced by the 4-bit flip-flop $f_{10}$. (f) Sets of flip-flops after merging.

their types in the library. Then, for each combination $\boldsymbol{n}$ in $\boldsymbol{T}$, we serially merge the flip-flops linked below the left child and the right child of $\boldsymbol{n}$ from leaves to root. Algorithm 3 shows the procedure to get a new flip-flop corresponding to the combination $n$. Based on its binary tree, we can find the combinations associated with the left child and right child of the root. Hence, the flip-flops in the lists, named $l_{\text {left }}$ and $l_{\text {right }}$, linked below the combinations of its left child and its right child are checked (see Lines 2 and 3). Then, for each flip-flop $f_{i}$ in $l_{\text {left }}$, the best flip-flop $f_{\text {best }}$ in $l_{\text {right }}$, which is the flip-flop that can be merged with $f_{i}$ with the smallest cost recorded in $c_{\text {best }}$, is picked. For each pair of flip-flops in the respective list, the combination cost [based on (5)] is computed if they can be merged and the pair with the smallest cost is chosen (see Lines 4-11). Finally, we add a new flip-flop $f^{\prime}$ in the list of the combination $n$ and remove the picked flip-flops which constitutes the $f^{\prime}$ (see Lines 12-14).

For example, given a library containing three types of flipflops (1-, 2-, and 4-bit), we first build a combination table $\boldsymbol{T}$ as shown in Fig. 12(a). In the beginning, the flip-flops with various types are, respectively, linked below $n_{1}, n_{2}$, and $n_{3}$ in 


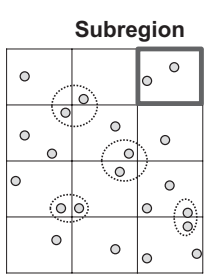

(a)

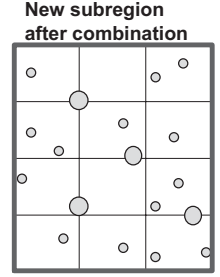

(b)
Fig. 13. Combination of flip-flops near subregion boundaries. (a) Result of replace flip-flops in each subregion. (b) Result of replace flip-flops in each new subregion which is obtained from combining twelve subregion in (a).

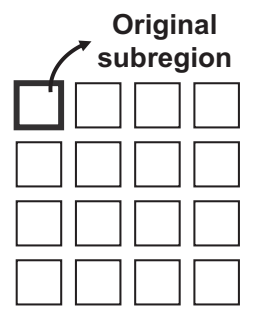

(a)

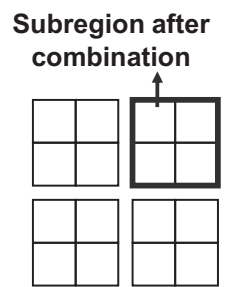

(b)

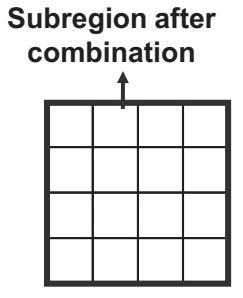

(c)
Fig. 14. Combination of subregions to a larger one. (a) Placement is originally partitioned into 16 subregions for replacement. (b) Subregion bounded by bold line is obtained from combining four neighboring subregions in (a). (c) Subregion bounded by bold line is obtained from combining four subregions in (b).

$\boldsymbol{T}$ according to their types. Suppose we want to form a flipflop in $n_{4}$, which needs two 1-bit flip-flops according to the combination table. Each pair of flip-flops in $n_{1}$ are selected and checked to see if they can be combined (note that they also have to satisfy the timing and capacity constraints described in Section II). If there are several possible choices, the pair with the smallest cost value is chosen to break the tie. In Fig. 12(a), $f_{1}$ and $f_{2}$ are chosen because their combination gains the smallest cost. Thus, we add a new node $f_{3}$ in the list below $n_{4}$, and then delete $f_{1}$ and $f_{2}$ from their original list [see Fig. 12(b)]. Similarly, $f_{4}$ and $f_{5}$ are combined to obtain a new flip-flop $f_{6}$, and the result is shown in Fig. 12(c). After all flip-flops in the combinations of 1-level trees $\left(n_{4}\right.$ and $\left.n_{5}\right)$ are obtained as shown in Fig. 12(d), we start to form the flip-flops in the combinations of 2-level trees $\left(n_{6}\right.$, and $\left.n_{7}\right)$. In Fig. 12(e), there exist some flip-flops in the lists below $n_{2}$ and $n_{4}$, and we will merge them to get flip-flops in $n_{6}$ and $n_{7}$, respectively. Suppose there is no overlap region between the couple of flipflops in $n_{2}$ and $n_{4}$. It fails to form a 4-bit flip-flop in $n_{6}$. Since the 2-bit flip-flops $f_{3}$ and $f_{6}$ are mergeable, we can combine them to obtain a 4-bit flip-flop $f_{10}$ in $n_{7}$. Finally, because there exists no couple of flip-flops that can be combined further, the procedure finishes as shown in Fig. 12(f).

If the available overlap region of two flip-flops exists, we can assign a new one to replace those flip-flops. Once there is sufficient space to place the new flip-flop in the available region, the algorithm will perform the replacement, and the new generated flip-flop will be placed in the grid that makes the wirelength between the flip-flop and its connected pins smallest. If the capacity constraint of the bin, $B_{k}$, which the grid belongs to will be violated after the new flip-flop is placed on that grid, we will search the bins near $B_{k}$ to find a new available grid for the new flip-flop. If none of bins which are overlapped with the available region of new flip-flop can satisfy the capacity constraint after the placement of new flip-flop, the program will stop the replacement of the two flip-flops.

3) Bottom-Up Flow of Subregion Combinations (Optional): As shown in Fig. 13(a), there may exist some flip-flops in the boundary of each subregion that cannot be replaced by any flip-flop in its subregion. However, these flip-flops may be merged with other flip-flops in neighboring subregions as shown in Fig. 13(b). Hence, to reduce power consumption further more, we can combine several subregions to obtain a larger subregion and perform the replacement again in the new subregion again. The procedure repeats until we cannot achieve any replacement in the new subregion. Fig. 14 gives an example for this hierarchical flow. As shown in Fig. 14(a), suppose we divide a chip into 16 subregions in the beginning. After the replacement of flip-flops is finished in each subregion, four subregions are combined to get a larger one as shown in Fig. 14(b). Suppose some flip-flops in new subregions still can be replaced by new flip-flops in other new subregions, we would combine four subregions in Fig. 14(b) to get a larger one as shown in Fig. 14(c) and perform the replacement in the new subregion again. As the procedure repeats in a higher level, the number of mergeable flip-flops gets fewer. However, it would spend much time to get little improvement for power saving. To consider this issue, there exists a trade-off between power saving and time consuming in our program.

4) De-Replace and Replace (Optional): Since the pseudo type is an intermediate type, which is used to enumerate all possible combinations in the combination table $\boldsymbol{T}$, we have to remove the flip-flops belonging to pseudo types. Thus, after the above procedures have been applied, we would perform de-replacement and replacement functions if there exists any flop-flops belonging to a pseudo type. For example, if there still exists a flip-flop, $f_{i}$, belonging to $n_{3}$ after replacements in Fig. 9(f), we have to de-replace $f_{i}$ into two flip-flops originally belongs to $n_{1}$. After de-replacing, we will do the replacements of flip-flops according to $\boldsymbol{T}$ without consideration of the combinations whose corresponding type is pseudo in $\boldsymbol{L}$.

\section{Computation COMPLEXity}

This section analyzes the timing complexity of this algorithm. The core is to continuously seek suitable combinations, and find the optimized solution among all possibilities. Hence, the timing complexity depends on the operation count of the function of deciding whether two flip-flops can combine together or not. For example, assume all flip-flops are of the same type, 1-bit flip-flop. In the beginning, each flip-flop will try to combine with all the other flip-flops. If the first flipflop finds the best solution, the two 1-bit flip-flops will form a 2-bit flip-flop and be removed from the list. Then, the second flip-flop will perform identical procedures. Let $N$ represent the number of flip-flops per circuit. For an exhaustive run for all the 1-bit cells, the timing complexity is $\mathrm{O}\left(N^{2}\right)$. If the 


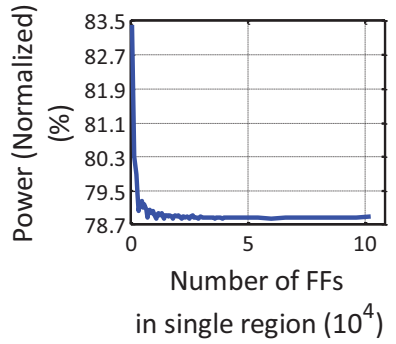

(a)

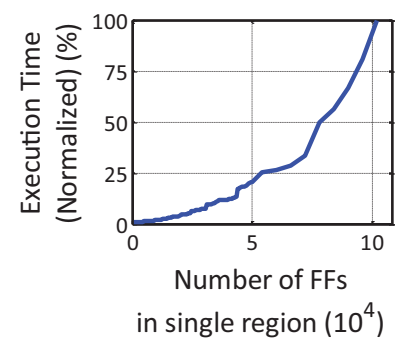

(b)
Fig. 15. (a) Influence of the region size on power. (b) Influence of the region size on execution time.

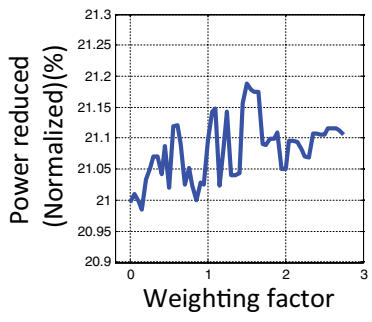

(a)

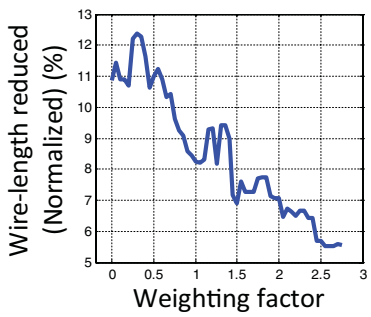

(b)
Fig. 16. (a) Influence of the weighting factor on power reduction. (b) Influence of the weighting factor on wirelength reduction.

largest flip-flop the library provided is $M$-bit, the size of the combination table is $\mathrm{O}\left(M \log _{2}(M)\right)$ when we use pseudo type flip-flops. The total timing complexity is $\mathrm{O}\left(M \log _{2}(M) \times N^{2}\right)$, equivalently equal to $\mathrm{O}\left(N^{2}\right)$ because the value of $M$ is much less than the value of $N$.

\section{EXPERIMENTAL RESULTS}

This section shows our experimental results. We implemented our algorithm in $\mathrm{C}++$ language, and all experiments were ran on workstation with a 3.33-GHz Intel Core i7-980X processor with 16-GB memory. Our experiment can be divided into two parts. In the first part, we compare our method with Chang et al. [6] and the results are shown in the first subsection. However, some conditions cannot be verified by their test cases. Thus, we provide another set of test cases and the experiment results are shown in the second subsection.

\section{A. Performance Comparison With Chang et al. [6]}

In this subsection, we first compare the experimental results with [6]. They used six test cases which were provided by Faraday corporation [7]. Table I shows the information of test cases. The numbers of flip-flops range from 98 to 169200 , and the available types (i.e., 1-, 2-, and 4-bit) of flip-flops in all cases are the same. Table I shows the number of flip-flops in each type in the initial condition.

In our algorithm, there exist two values which would affect our results: the first one is the dimension of a subregion since we would partition a chip into several subregions. The second one is the parameter used in the cost function of (5). Thus, we first do some experiments to explore better values for these two parameters. The results for comparisons with [6] will be shown in the last part of this subsection.
TABLE I

INDUSTRY BENCHMARK CIRCUITS

\begin{tabular}{|c|c|c|c|}
\hline Circuit & $\begin{array}{c}\text { Number of } \\
\text { 1-bit FFs }\end{array}$ & $\begin{array}{c}\text { Number of } \\
\text { 2-bit FFs }\end{array}$ & $\begin{array}{c}\text { Number of } \\
\text { 4-bit FFs }\end{array}$ \\
\hline $\boldsymbol{c 1}$ & 76 & 22 & 0 \\
\hline $\boldsymbol{c 2}$ & 366 & 57 & 0 \\
\hline $\boldsymbol{c 3}$ & 1464 & 228 & 0 \\
\hline $\boldsymbol{c 4}$ & 4378 & 751 & 0 \\
\hline $\boldsymbol{c 5}$ & 9150 & 1425 & 0 \\
\hline $\boldsymbol{c 6}$ & 146400 & 22800 & 0 \\
\hline
\end{tabular}

TABLE II

EXPERIMENTAL RESULTS OF [6] AND OUR APPROACH

\begin{tabular}{|c|c|c|c|c|c|c|}
\hline \multirow{2}{*}{ Circuit } & \multicolumn{3}{|c|}{ Approach in [6] } & \multicolumn{3}{|c|}{ Our approach } \\
\hline & $\begin{array}{c}\text { PR_Ratio } \\
(\%)\end{array}$ & $\begin{array}{c}\text { WR_Ratio } \\
(\%)\end{array}$ & $\begin{array}{c}\text { Times } \\
\text { (s) }\end{array}$ & $\begin{array}{c}\text { PR_Ratio } \\
(\%)\end{array}$ & $\begin{array}{c}\text { WR_Ratio } \\
(\%)\end{array}$ & $\begin{array}{c}\text { Times } \\
\text { (s) }\end{array}$ \\
\hline$c 1$ & 14.8 & 0.917 & 0.01 & 15.9 & 0.928 & 0.07 \\
\hline$c 2$ & 16.9 & 0.947 & 0.04 & 18.0 & 0.934 & 0.12 \\
\hline$c 3$ & 17.1 & 0.948 & 0.10 & 17.8 & 0.928 & 0.24 \\
\hline$c 4$ & 16.8 & 0.945 & 0.28 & 17.6 & 0.932 & 0.84 \\
\hline$c 5$ & 17.1 & 0.949 & 0.60 & 17.8 & 0.936 & 1.51 \\
\hline$c 6$ & 17.2 & 0.949 & 78.92 & 17.9 & 0.938 & 30.43 \\
\hline Comp. & 0.95 & 1.01 & 2.41 & 1.00 & 1.00 & 1.00 \\
\hline
\end{tabular}

1) Influence of Region Size on Performance: In this part, we first determine a suitable size for each subregion during partitioning. Since the execution time is actually dominated by the average number of flip-flops included in a subregion, we use the number of flip-flops in a single subregion to represent the size of a subregion, which can be obtained from multiplying the number of bins in a subregion by the average number of flip-flops in a bin. Fig. 15 shows the simulation results using the circuit $\boldsymbol{c} 6$ in Table I. We sweep the number of flip-flops included in a subregion to observe its effect on power consumption and execution time. The $y$-axis in Fig. 15(a) and (b), respectively, represent the power reduction and timing improvement ratios relative to the size of a subregion. While a subregion gets larger, the execution time becomes longer. However, the power consumption does not decrease proportionally. On the contrary, if the subregion size becomes very small, the power consumption will increase significantly. To balance execution time and power consumption, we select 600 as the number of flip-flops in a single subregion (the normalized power and execution time are about $83 \%$ and $0.8 \%$ if the number of flip-flops in a single subregion is 600 in Fig. 15).

2) Influence of Weighting Factor $\alpha$ on Performance: Since the parameter $\alpha$ used by (5) (see Section III-C.2) would affect our results, it is necessary to find a suitable value for getting better results. Similarly, we use circuit $\mathbf{c 6}$ to test our program, and the simulation result is shown in Fig. 16. In this experiment, we sweep $\alpha$ from 0 to 3 to get the data of power consumption and wirelength. The $y$-axis in Fig. 16(a) and (b) respectively represents the wirelength reduction ratio and the power reduction ratio. While the value of $\alpha$ becomes 
TABLE III

EXPERIMENTAL RESUlts UndER DifFERENT CONDITIONS

\begin{tabular}{|c|c|c|c|c|c|}
\hline & Case 1 & Case 2 & Case 3 & Case 4 & Case 5 \\
\hline Library & $1,2,4$ & $1,2,4,4,8$ & $1,2,4,6,13$ & $1,2,4,8$ & $1,2,4,8$ \\
\hline Flip-flop number & 120 & 953 & 5524 & 60000 & 1728000 \\
\hline Power $_{\text {ori }}$ (unit $10^{3}$ ) & 12 & 95 & 552 & 6000 & 172800 \\
\hline Power $_{\text {merged }}$ (unit $10^{3}$ ) & 9 & 67 & 430 & 4208 & 136509 \\
\hline PR_Ratio (\%) & 20.97 & 28.80 & 22.11 & 29.87 & 21.00 \\
\hline $\mathbf{W L}_{\text {ori }}\left(\right.$ unit $10^{3}$ ) & 83 & 577 & 3563 & 53625 & 1199304 \\
\hline$W L_{\text {merged }}\left(\right.$ unit $10^{3}$ ) & 71 & 506 & 2189 & 31008 & 1068961 \\
\hline WR_Ratio (\%) & 85.62 & 87.77 & 61.44 & 57.82 & 89.13 \\
\hline Times (s) & 0.08 & 0.24 & 1.07 & 36.7 & 2377 \\
\hline Times of parser & 0.07 & 0.15 & 0.29 & 3.8 & 2153 \\
\hline
\end{tabular}

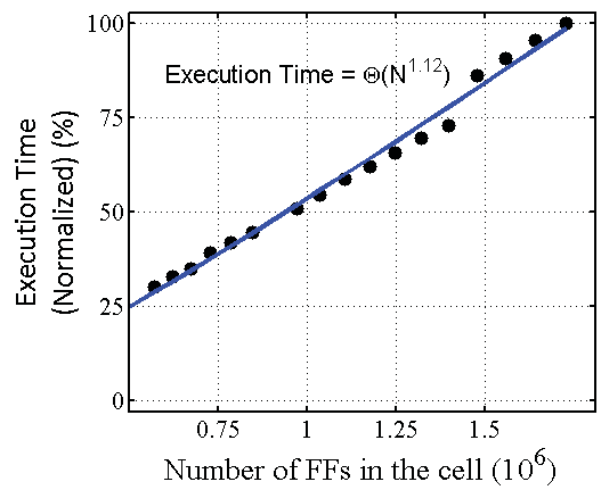

Fig. 17. Average computational complexity of our algorithm.

larger, the power reduction ratio gets larger. If $\alpha$ is close to 0 , the wirelength reduction ratio will be better than the power reduction ratio. To balance wirelength reduction and power reduction, we use the curves to select a suitable value for $\alpha$. Because the variation of $\alpha$ has the more apparent effect on wirelength reduction than power reduction, the value of $\alpha$ close to 0 is preferred. In the following experiments, we select 0.1 as the value of $\alpha$.

3) Comparison Results: The comparison results between [6] and our approach are listed in Table II. Column 1 lists the names of benchmark circuits. In [6], their algorithm was implemented on $2.66-\mathrm{GHz}$ Intel i7 PC under the Linux operation system, and our algorithm was implemented on a 3.33-GHz Intel Core i7-980X processor with 16-GB memory. In Table II, we compare the results of PR_Ratio, WR_Ratio and execution times with [6]. The comparison results are listed in row 8. The values PR_Ratio and WR_Ratio can be computed by the following equations:

$$
\begin{gathered}
\text { PR_Ratio }(\%)=\frac{\text { power }_{\text {original }}-\text { power }_{\text {merged }}}{\text { power }_{\text {original }}} \cdot 100 \% \\
\text { WR_Ratio(\%) }=\frac{\text { wire_length }_{\text {merged }}}{\text { wire_length }_{\text {original }}} \cdot 100 \%
\end{gathered}
$$

where the power $r_{\text {merged }}$ and wire_length ${ }_{\text {merged }}$ are the measured power and wirelength after the program is applied, and the power $_{\text {original }}$ and wire_length ${ }_{\text {original }}$ are the measured power and wirelength of the original test case. As shown in Table II,

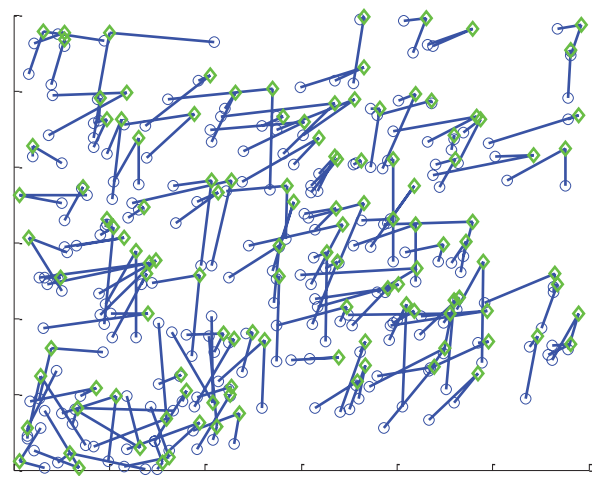

Fig. 18. Distribution of flip-flops in the original design (120 flip-flops, power $=12000$, wirelength $=83285$ ).

our results of PR_Ratio, WR_Ratio and execution time are all better than the results in [6]. Our execution time of cases with number of flip-flops smaller than about 10000 is larger than [6], because we have to spend additional time to build the combination table. However, with the help of the combination table, our experimental results of the execution time of $c 6$ (about 170000 flip-flops) is much less than [6].

\section{B. Average-Case Performance}

In this subsection, we provide another set of cases supported by [9] to test our program. The content of test circuits and experimental results are shown in Table III. Compared to the cases in Table I, the available types of flip-flops are different from Cases 1 to 5 . Case 5 is the largest circuit of about 1700000 flip-flops. Because the execution time is dominated by the number of flip-flops in the circuit, Case 5 is applied to help to demonstrate the efficiency and robust of our algorithm. Row 1 in the table lists all test cases and row 2 shows types of different flip-flops that can be used in each test case. Rows 3 and 4 respectively, show numbers of flip-flops and total power consumption in original test cases. After some flip-flops are replaced by our algorithm, the power consumption of each design is shown in row 5, and row 6 computes the ratio of power reduction by our algorithm, which is denoted by PR_Ratio. From rows 7 to 9 , it shows the wirelength reduction by our algorithm. Rows 7 and 8 show the original wirelength and the wirelength after our program is applied. Finally, the 


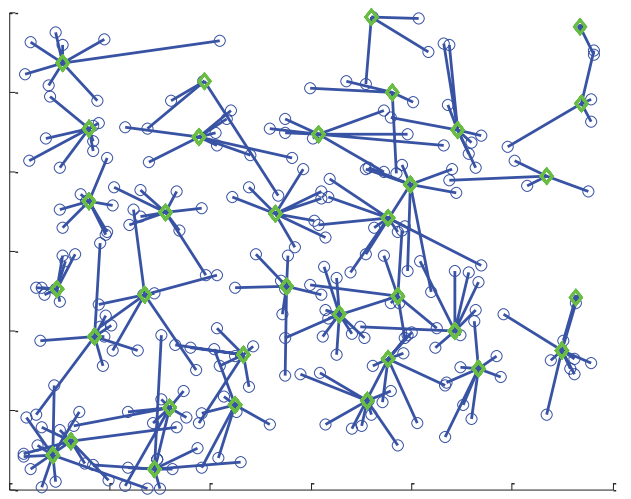

Fig. 19. Resulting distribution of flip-flops (34 flip-flops, power $=9484$, wirelength $=71304$ )

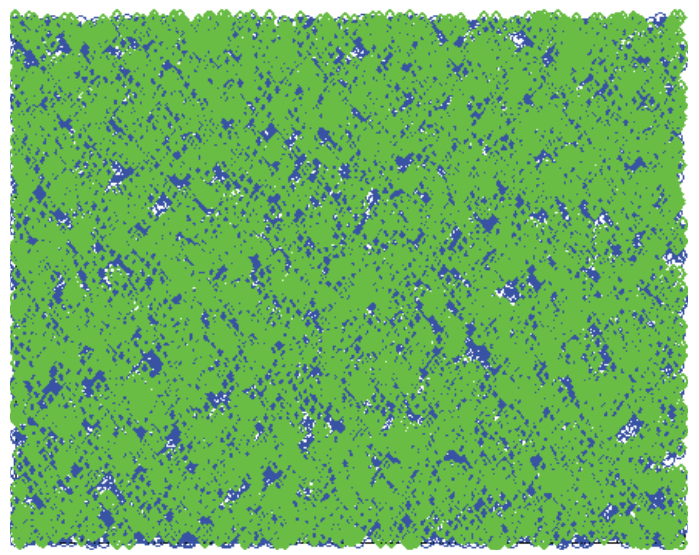

Fig. 20. Distribution of flip-flops in the original design. (5524 flip-flops, power $=552400$, wirelength $=3562985$ ).

ratio of wirelength reduction, which is denoted by WR_Ratio, is shown in row 9 .

The values of PR_Ratio in all cases are between 20 and 30 . Besides, the wirelength are less than the original circuit in all cases, and the best value of WR_Ratio can achieve $42.18 \%$ improvement. Row 10 shows the execution time of each case. Because of the long execution time of parser, we show the execution time of parser in row 11.

Fig. 17 displays the curve of the execution time with respect to various flip-flop numbers in a circuit. The test cases are obtained by duplicating Case 1 various times. The $x$-axis represents the number of flip-flops, and the $y$-axis denotes the percentage of a execution time compared with the longest execution time. As the number of flip-flops increases, the execution time of parser will be longer than execution time which does not include parser. For this reason, the execution time in Fig. 17 does not include the execution time of parser. The largest case, which contains about 1700000 flip-flops, takes the longest execution time (about $10 \mathrm{~min}$ ). According to Fig. 17, it shows that the timing complexity of our algorithm is $\mathrm{O}\left(N^{1.12}\right)$ instead of $\mathrm{O}\left(N^{2}\right)$.

Figs. 18 and 19 show the original distribution of flip-flops and the resulting distribution of flip-flops after applying our program. In the figures, flip-flops are denoted by green circles and pins by blue circles. Blue lines represent the wires that

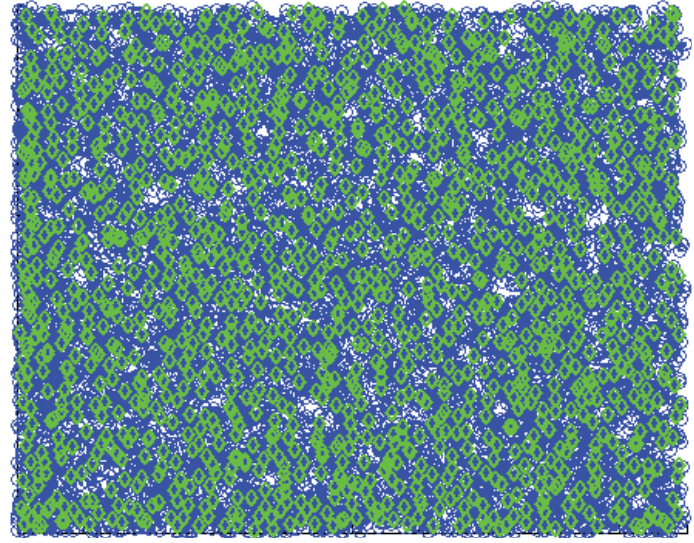

Fig. 21. Resulting distribution of flip-flops. (1378 flip-flops, power $=$ 430260 , wirelength $=2189215$ ).

connect pins and flip-flops. In Fig. 18, there are 120 1-bit flip-flops and 240 pins in the original circuit in Case 1. After applying our program, there only exist 27 4-bit flip-flops, five 2-bit flip-flops and two 1-bit flip-flops in the new design shown in Fig. 19. In Fig. 20, there exist 5524 2-bit flip-flops and 11048 pins in the original circuit in Case 3. There only exist two 6-bit, 1284 4-bit, 34 2-bit, and eight 1-bit flip-flops for the new circuit shown in Fig. 21 after applying our program.

\section{CONCLUSION}

This paper has proposed an algorithm for flip-flop replacement for power reduction in digital integrated circuit design. The procedure of flip-flop replacements is depending on the combination table, which records the relationships among the flip-flop types. The concept of pseudo type is introduced to help to enumerate all possible combinations in the combination table. By the guidelines of replacements from the combination table, the impossible combinations of flip-flops will not be considered that decreases execution time. Besides power reduction, the objective of minimizing the total wirelength also be considered to the cost function. The experimental results show that our algorithm can achieve a balance between power reduction and wirelength reduction. Moreover, even for the largest case which contains about 1700000 flip-flops, our algorithm can maintain the performance of power and wirelength reduction in the reasonable processing time.

\section{REFERENCES}

[1] P. Gronowski, W. J. Bowhill, R. P. Preston, M. K. Gowan, and R. L. Allmon, "High-performance microprocessor design," IEEE J. Solid-State Circuits, vol. 33, no. 5, pp. 676-686, May 1998.

[2] W. Hou, D. Liu, and P.-H. Ho, "Automatic register banking for lowpower clock trees," in Proc. Quality Electron. Design, San Jose, CA, Mar. 2009, pp. 647-652.

[3] D. Duarte, V. Narayanan, and M. J. Irwin, "Impact of technology scaling in the clock power," in Proc. IEEE VLSI Comput. Soc. Annu. Symp., Pittsburgh, PA, Apr. 2002, pp. 52-57.

[4] H. Kawagachi and T. Sakurai, "A reduced clock-swing flip-flop (RCSFF) for 63\% clock power reduction," in VLSI Circuits Dig. Tech. Papers Symp., Jun. 1997, pp. 97-98.

[5] Y. Cheon, P.-H. Ho, A. B. Kahng, S. Reda, and Q. Wang, "Power-aware placement," in Proc. Design Autom. Conf., Jun. 2005, pp. 795-800. 
[6] Y.-T. Chang, C.-C. Hsu, P.-H. Lin, Y.-W. Tsai, and S.-F. Chen, "Post-placement power optimization with multi-bit flip-flops," in Proc. IEEE/ACM Comput.-Aided Design Int. Conf., San Jose, CA, Nov. 2010, pp. 218-223.

[7] Faraday Technology Corporation [Online]. Available: http://www. faraday-tech.com/index.html

[8] C. Bron and J. Kerbosch, "Algorithm 457: Finding all cliques of an undirected graph," ACM Commun., vol. 16, no. 9, pp. 575-577, 1973.

[9] CAD Contest of Taiwan [Online]. Available: http://cad_contest.cs. nctu.edu.tw/cad11

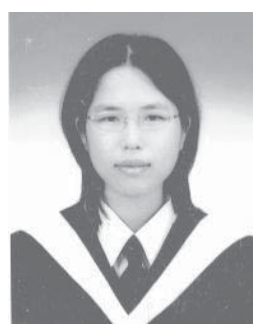

Ya-Ting Shyu received the M.S. degree in electrical engineering from National Cheng Kung University (NCKU), Tainan, Taiwan, in 2008, where she is pursuing the $\mathrm{Ph} . \mathrm{D}$. degree in electronic engineering.

Her current research interests include integrated circuit design, design automation for analog, and mixed-signal circuits.

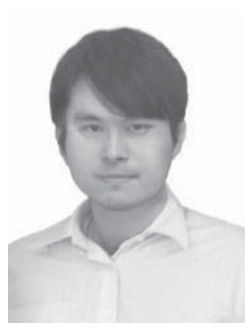

Jai-Ming Lin received the B.S., M.S., and Ph.D degrees from National Chiao Tung University, Hsinchu, Taiwan, in 1996, 1998, and 2002, respectively, all in computer science.

He was an Assistant Project Leader with the CAD Team, Realtek Corporation, Hsinchu, from 2002 to 2007. He is currently an Assistant Professor with the Department of Electrical Engineering, National Cheng Kung University, Tainan, Taiwan. His current research interests include floorplan, placement, routing, and clock tree synthesis.

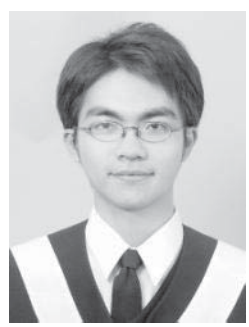

Chun-Po Huang was born in Tainan, Taiwan, in 1986. He received the B.S. degree in electrical engineering from National Cheng Kung University, Tainan, Taiwan, in 2008, where he is currently pursuing the Ph.D. degree in electronic engineering.

His current research interests include design automation for high-speed and low-power analogto-digital converters.

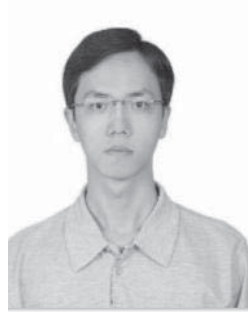

Cheng-Wu Lin received the M.S. degree in electrical engineering from National Cheng Kung University (NCKU), Tainan, Taiwan, in 2006, where he is currently pursuing the Ph.D. degree in electronic engineering.

His current research interests include integrated circuit design, design automation for analog, and mixed-signal circuits.

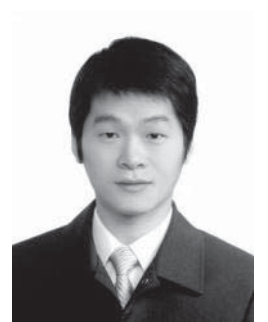

Ying-Zu Lin received the B.S. and M.S. degrees in electrical engineering and the Ph.D. degree from National Cheng Kung University, Tainan, Taiwan, in 2003, 2005, and 2010, respectively.

$\mathrm{He}$ is currently with Novatek, Hsinchu, Taiwan, a Senior Engineer, where he is working on highspeed interfaces and analog circuits for advanced display systems. His current research interests include analog/mixed-signal circuits, analog-todigital converters, and high-speed interface circuits.

Dr. Lin was the recipient of the Excellent Award in the master thesis contest held by the Mixed-Signal and Radio-Frequency Consortium, Taiwan, in 2005, the Best Paper Award of the VLSI Design/Computer-Aided Design Symposium, Taiwan, in 2008, and the Taiwan Semiconductor Manufacturing Company Outstanding Student Research Award. He received third prize in the Acer Dragon Award for Excellence. He was the recipient of the MediaTek Fellowship in 2009, the Best Paper Award from the Institute of Electronics, Information, and Communication Engineers, and the Best Ph.D. Award from the IEEE Tainan Section in 2010. He was a co-recipient of the Gold Award in Macronix Golden Silicon Design Contests in 2010. He was a recipient of the International Solid State Circuits Conference/Design Automation Conference Student Design Contest in 2011, the Chip Implementation Center Outstanding Chip Design Award (Best Design), and the International Symposium of Integrated Circuits Chip Design Competition.

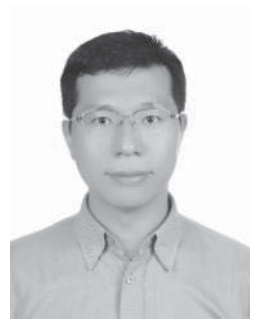

Soon-Jyh Chang (M'03) was born in Tainan, Taiwan, in 1969. He received the B.S. degree in electrical engineering from National Central University, Jhongli, Taiwan, in 1991, and the M.S. and $\mathrm{Ph} . \mathrm{D}$. degrees in electronic engineering from National Chiao Tung University, Hsinchu, Taiwan, in 1996 and 2002, respectively.

$\mathrm{He}$ has been with the Department of Electrical Engineering, National Cheng Kung University, Tainan, since 2003, where he is currently a Professor and the Director of the Electrical Laboratories since 2011. He has authored or co-authored over 100 technical papers and 7 patents. His current research interests include design, testing, and design automation for analog and mixed-signal circuits.

Dr. Chang has been serving as the Chair of the IEEE Solid-State Circuits Society Tainan Chapter since 2009. He was the Technical Program CoChair of the IEEE Institute for Sustainable Nanoelectronics in 2010, and the Committee Member of the IEEE Asian Test Symposium in 2009, Asia and South Pacific Design Automation Conference in 2010, the VLSI-Design, Automation, and Test in 2009, 2010, and 2012, and the Asian Solid-State Circuits Conference in 2009 and 2011. He was a recipient and co-recipient of many technical awards, including the Greatest Achievement Award from the National Science Council, Taiwan, in 2007, the Chip Implementation Center Outstanding Chip Award in 2008, 2011, and 2012, the Best Paper Award of VLSI Design/Computer-Aided Design Symposium, Taiwan, in 2009 and 2010, the Best Paper Award of the Institute of Electronics, Information and Communication Engineers in 2010, the Gold Prize of the Macronix Golden Silicon Award in 2010, the Best GOLD Member Award from the IEEE Tainan Section in 2010, the International Solid State Circuits Conference/Design Automation Conference Student Design Contest in 2011, and the International Symposium on Integrated Circuits Chip Design Competition in 2011. 